\title{
Briófitas de um remanescente de Mata Atlântica no Município de Ubajara, CE, Brasil
}

\author{
Samylia Mayra da Costa Siqueira ${ }^{1,3}$, Patrícia Silva Costa ${ }^{1}$, Elnatan Bezerra de Souza ${ }^{1}$ e \\ Hermeson Cassiano de Oliveira ${ }^{2}$
}

Recebido: 8.06.2011; aceito: 24.11.2011

\begin{abstract}
Bryophytes from a remnant of Atlantic Forest in the municipality of Ubajara, Ceará State, Brazil). This paper presents the survey of the bryophytes of the remnant of Atlantic Forest, in the municipality of Ubajara, Ceará State, Brazil. Were recorded 102 species in 53 genera and 29 families. Lejeuneaceae and Fissidentaceae were the families with larger specific richness. Aphanolejeunea kunertiana Steph., Groutiella apiculata (Hook.) H.A. Crum \& Steere, Lophocolea liebmanniana Gottsche, Orthostichopsis praetermissa W.R. Buck, and O. tortipilis (Müll. Hal.) Broth. are recorded for the first time to Ceará State.
\end{abstract}

Key words: hornworts, Ibiapaba, liverworts, mosses

RESUMO - (Briófitas de um remanescente de Mata Atlântica no Município de Ubajara, CE, Brasil). Este trabalho consiste no levantamento de briófitas em um remanescente de Mata Atlântica no Município de Ubajara, Brasil. Foram registradas 102 espécies pertencentes a 53 gêneros e 29 famílias. Lejeuneaceae e Fissidentaceae foram as famílias com maior riqueza específica. Aphanolejeunea kunertiana Steph., Groutiella apiculata (Hook.) H.A. Crum \& Steere, Lophocolea liebmanniana Gottsche, Orthostichopsis praetermissa W.R. Buck e Orthostichopsis tortipilis (Müll. Hal.) Broth. são citadas pela primeira vez para o Estado do Ceará.

Palavras-chave: antóceros, hepáticas, Ibiapaba, musgos

\section{Introdução}

A Mata Atlântica destaca-se por ter um dos complexos vegetacionais mais singulares, alta diversidade de espécies e elevado nível de endemismo (Mori et al. 1981, Fonseca 1985). Esse bioma vem passando por extração não sustentável de espécies de plantas e madeira há um longo período, fazendo com que restem poucas áreas preservadas (Galindo-Leal \& Câmara 2005). Dados atuais indicam que restam cerca de $11 \%$ da vegetação original (Ribeiro et al. 2009). No Nordeste brasileiro as áreas estão fragmentadas em pequenas manchas de matas, cercadas por extensas plantações de cana-de-açúcar ou áreas urbanas (Pereira \& Alves 2006). Em estudos recentes, Costa (2009) considerou a Mata Atlântica como sendo a mais rica região do país em termos de diversidade de briófitas, com $71 \%$ das espécies reconhecidas para o Brasil.
Os estudos de briófitas para a Mata Atlântica são conhecidos principalmente pelos trabalhos de: Germano \& Pôrto (1996, 1998), Oliveira-e-Silva et al. (2002), Visnadi (2002, 2005), Costa \& Silva (2003), Costa et al. (2005), Valente \& Pôrto (2006), Peralta \& Athayde Filho (2008), Santos \& Costa (2008), Costa \& Santos (2009), Valente et al. (2009) e Vilas Bôas-Bastos \& Bastos (2009).

O conhecimento sobre a brioflora do Ceará tem avançado principalmente na última década. Brito \& Pôrto (2000) elaboraram uma chave para as famílias de briófitas ocorrentes no Estado e afirmaram que os encraves úmidos e subúmidos estudados, constituem remanescentes de Mata Atlântica. Yano \& Pôrto (2006) citaram 155 espécies, das quais 130 constituíram novas ocorrências para o Ceará; e Yano et al. (2009) registraram 68 espécies para o Estado. Para o Planalto da Ibiapaba, destacam-se as contribuições de Oliveira

1. Universidade Estadual Vale do Acaraú, Coordenação de Biologia, Av. da Universidade 850, Campus Betânia, 62040-370 Sobral, CE, Brasil

2. Universidade Estadual de Feira de Santana, Departamento de Ciências Biológicas, Programa de Pós-Graduação em Botânica, Av. Transnordestina s/n, 44036-900 Feira de Santana, BA, Brasil

3. Autor para correspondência: samyliamayra@hotmail.com 
\& Bastos (2009a, b, 2010a, b), cujos levantamentos resultaram em um total de 71 espécies para a região. A única referência sobre a brioflora do Município de Ubajara é o trabalho de Oliveira \& Alves (2007), no qual foram registradas 35 novas ocorrências para o Estado do Ceará.

Dessa forma, o presente trabalho teve como objetivo dar prosseguimento ao estudo da brioflora do Estado do Ceará, por meio do levantamento dos táxons ocorrentes em um remanescente de Mata Atlântica no Município de Ubajara.

\section{Material e métodos}

O fragmento de mata estudado está situado no Sítio São Luís, Município de Ubajara, Estado do Ceará, nas coordenadas $3^{\circ} 47^{\prime} 53^{\prime \prime} \mathrm{S}$ e $40^{\circ} 54^{\prime} 19^{\prime \prime} \mathrm{W}$. Ocupa uma área de aproximadamente 32 ha, com altitudes variando de 700 a $900 \mathrm{~m}$. As precipitações anuais oscilam entre 1.350 e $1.750 \mathrm{~mm}$ (Bezerra et al. 1989) e os solos são do tipo Latossolo Vermelho Amarelo Distrófico, com estrutura física muito favorável à erosão (Leite \& Marques 1989). A área de estudo faz parte do Planalto da Ibiapaba, um relevo em forma de cuesta (Souza 1989), com a vertente oriental, escarpada e íngreme, exposta aos efeitos das chuvas orográficas e do orvalho produzido pelo nevoeiro, condicionantes principais da ocorrência de matas úmidas de altitude, denominada por Figueiredo (1989) como Floresta Subperenifólia Tropical Plúvio-Nebular, sendo considerada como fragmento de Floresta Atlântica (Fernandes 1990). Vale ressaltar, que a porção mais úmida do Planalto da Ibiapaba, denominada Ibiapaba Norte, é uma das áreas consideradas de extrema importância biológica para a conservação da biodiversidade (Tabarelli \& Cardoso-Silva 2002).

As coletas foram realizadas no período de 2004 a 2011, por meio de caminhadas pelas trilhas do sítio, considerando os períodos úmidos e secos. A classificação quanto ao tipo de substrato colonizado seguiu Robbins (1952), segundo o qual a amostra coletada pode ser considerada corticícola (tronco vivo), epíxila (tronco em decomposição), rupícola (materiais em rocha), terrícola (em solo) ou epífila (em superfície de folha).

Para identificação das amostras foram utilizados os trabalhos de Hässel-de-Menéndez (1962), Bischler (1964), Florschütz (1964), Hell (1969), Schuster (1980, 1992), Churchill (1988), Inoue (1989), Yuzawa (1991), Gradstein (1994), Sharp et al. (1994), Buck
(1998), Heinrichs et al. (1998), Lemos-Michel \& Yano (1998), Heinrichs \& Gradstein (2000), Oliveira-e-Silva \& Yano (2000), Reiner-Drehwald (2000), Gradstein et al. (2001), Allen (2002), Ilkiu-Borges \& Lisboa (2002), Visnadi (2002), Gradstein \& Costa (2003), Yano \& Peralta (2007), Zartman \& Ilkiu-Borges (2007), Pursell (2008) e Oliveira \& Bastos (2009a, b, 2010a, b). Além disso, foram realizadas consultas a especialistas e análises das coleções dos Herbários HUVA e HUEFS. Os sistemas de classificação adotados foram Crandall-Stotler et al. (2009) para Marchantiophyta, Renzaglia et al. (2008) para Anthocerotophyta e Goffinet et al. (2008) para Bryophyta.

A coleta e preservação das amostras basearam-se em Yano (1984a). A distribuição geográfica foi fundamentada nos catálogos de Yano (1981, 1984b, 1989, 1995, 2006, 2008, 2010).

As amostras foram incorporadas aos acervos dos Herbários Professor Francisco José de Abreu Matos (HUVA) da Universidade Estadual Vale do Acaraú, Herbário Alexandre Leal Costa (ALCB) da Universidade Federal da Bahia e Herbário da Universidade Estadual de Feira de Santana (HUEFS).

\section{Resultados e Discussão}

No Sítio São Luís a brioflora está representada por 29 famílias, 53 gêneros e 102 espécies (tabela 1). A família mais representativa foi Lejeuneaceae com 23 espécies, correspondendo a $22,5 \%$ do total, seguida de Fissidentaceae com 12 espécies, Plagiochilaceae e Sematophyllaceae com seis. Em estudos similares para Mata Atlântica como os de Valente \& Pôrto (2006) e Santos \& Costa (2008), essa família também aparece com maior riqueza florística, com 53 e 20\%, respectivamente. Aphanolejeunea kunertiana Steph., Groutiella apiculata (Hook.) H.A. Crum \& Steere, Lophocolea liebmanniana Gottsche, Orthostichopsis praetermissa W.R. Buck e O. tortipilis (Müll. Hal.) Broth. são novas citações para o Estado do Ceará.

Das 196 amostras analisadas, 55\% são de musgos, sendo Fissidens prionodes Mont., Henicodium geniculatum (Mitt.) W.R. Buck, Orthostichopsis praetermissa W.R. Buck e Sematophyllum subpinnatum (Brid.) E. Britton as espécies mais coletadas. Entre as hepáticas, Metzgeria furcata (L.) Dumort. e Plagiochila martiana (Nees) Lindenb. foram as espécies com maior quantidade de amostras. Os antóceros correspondem à $2 \%$ da amostragem do sítio. 
Tabela 1. Lista das espécies de briófitas do Sítio São Luís, Ubajara, CE, Brasil, com dados dos tipos de substrato. C: corticícolo. EF: epifilo. EX: epíxilo. R: rupícolo. T: terrícolo. As novas ocorrências para o Estado do Ceará estão assinaladas com um asterisco $\left(^{*}\right)$. Ao lado de cada divisão e família entre parênteses, estão indicados os números de famílias, gêneros e espécies.

Table 1. List of species of bryophytes of Sítio São Luís, Ubajara, Ceará State, Brazil, with substrate data. C: corticicolous. EF: epiphyllous. EX: epixilous. R: rupicolous. T: terricolous. The new records for the Ceará State are marked with an asterisk (*). Next to each family and division parentheses are indicated the numbers of families, genera and species.

\begin{tabular}{|c|c|c|c|}
\hline Filo/Família/Espécie & $\begin{array}{c}\text { Tipo de } \\
\text { substrato }\end{array}$ & $\begin{array}{c}\text { Distribuição geográfica } \\
\text { no Brasil }\end{array}$ & Voucher \\
\hline
\end{tabular}

ANTHOCEROTOPHYTA (2/2/2)

Anthocerotaceae (1/1)

Anthoceros punctatus L.

R AM, BA, CE, RJ, RS e SP

H.C. Oliveira 148, 150 HUVA

Notothyladaceae (1/1)

Notothylas orbicularis (Schwein.) Sull.

T $\quad \mathrm{CE}, \mathrm{GO}$ e PE

H.C. Oliveira 146, 159

HUVA

BRYOPHYTA (17/29/58)

Bartramiaceae (1/5)

Philonotis cernua (Wilson) D.G.

Griff. \& W.R. Buck

P. elongata (Dism.) H.A. Crum \&

Steere

P. hastata (Duby in Moritzi) Wijk

\& Moritzi

P. longiseta (Michx.) E. Britton.

P. uncinata (Schwägr.) Brid.

CE, DF, GO, MT, RJ, RS, SE

$\mathrm{C} / \mathrm{T}$

H.C. Oliveira 172

S.M.C. Siqueira 99

HUVA

R/T AM, CE, PB, PR e SP

E.B. Souza 1807

M.D. Silva 48

HUVA

H.C. Oliveira 444 HUEFS

R AM, CE, GO, MG, MS, MT,

PA, PR, RJ, RO, RS e SP

S.M.C. Siqueira $86 \quad$ HUVA

$\mathrm{AC}, \mathrm{AM}, \mathrm{BA}, \mathrm{CE}, \mathrm{DF}, \mathrm{ES}$, GO, MG, MS, MT, PA, PB,

T PE, PI, PR, RJ, RO, RS, SC,

H.C. Oliveira 88

HUVA

SP e TO

Brachythecyaceae (2/4)

Squamidium brasiliense (Hornsch.)

Broth.

BA, CE, ES, MG, PE, PR, RJ,

C

RS, SC e SP

S. leucotrichum (Taylor) Broth.

C/EX

AC, AL, AM, BA, CE, ES, PA,

RJ, RS, SC e SP

C

AP, CE, GO, MG, PE, PR, RJ, RS, SC e SP

AC, AL, AM, AP, BA, CE, ES,

C GO, MG, MS, MT, PA, PE,

PR, RJ, RO, RR, RS, SC e SP

C.J.P. Bastos 5357

S.B.V. Bôas-Bastos 2517, 2531, 2538

S.M.C. Siqueira 34

HUVA

M.D. Silva 20

HUVA

Manuel

Bryaceae (1/3)

Bryum cellulare Hook.

B. leptocladon Sull.

B. limbatum Müll. Hal.

$\mathrm{T}$

CE, MG e RS

H.C. Oliveira 169

HUVA

$\mathrm{T} \quad \mathrm{BA}, \mathrm{CE}$ e GO

H.C. Oliveira 164

HUVA

$\mathrm{T}$

BA, CE, ES, MG, PR, RJ, RS, SC e SP

H.C. Oliveira 98

HUVA

Calymperaceae (3/5) 
Tabela 1 (continuação)

\begin{tabular}{|c|c|c|c|c|}
\hline Filo/Família/Espécie & $\begin{array}{l}\text { Tipo de } \\
\text { substrato }\end{array}$ & $\begin{array}{c}\text { Distribuição geográfica } \\
\text { no Brasil }\end{array}$ & Voucher & Herbário \\
\hline Calymperes erosum Müll. Hal. & $\mathrm{C}$ & $\begin{array}{l}\text { AC, AM, AP, BA, CE, ES, } \\
\text { GO, MG, MT, PA, PB, PE, PI, } \\
\text { PR, RJ, RN, RO, RR, SE, SP } \\
\text { e TO }\end{array}$ & S.M.C. Siqueira 12 & HUVA \\
\hline C. palisotii Schwägr. & $\mathrm{R}$ & $\begin{array}{l}\text { AL, AM, AP, BA, CE, ES, } \\
\text { GO, MT, PA, PB, PE, PI, PR, } \\
\text { RJ, RN, RO, RR, SE, SP e TO }\end{array}$ & H.C. Oliveira 189 & HUVA \\
\hline Octoblepharum albidum Hedw. & $\mathrm{C}$ & $\begin{array}{l}\mathrm{AC}, \mathrm{AL}, \mathrm{AM}, \mathrm{AP}, \mathrm{BA}, \mathrm{CE} \\
\mathrm{DF}, \mathrm{ES}, \mathrm{GO}, \mathrm{MA}, \mathrm{MG}, \mathrm{MS}\end{array}$ & $\begin{array}{l}\text { H.C. Oliveira 18, 168, } \\
180\end{array}$ & HUVA \\
\hline & & $\begin{array}{l}\text { MT, PA, PB, PE, PI, PR, RJ, } \\
\text { RN, RO, RR, RS, SC, SE, SP } \\
\text { e TO. }\end{array}$ & & \\
\hline Syrrhopodon parasiticus (Brid.) Besch. & $\mathrm{C} / \mathrm{EX}$ & $\begin{array}{l}\text { AC, AM, BA, CE, DF, ES, } \\
\text { GO, MG, MS, MT, PA, PE, } \\
\text { PR, RJ, RO, RR, RS, SC, SP } \\
\text { e TO }\end{array}$ & S.M.C. Siqueira 70, 183 & HUVA \\
\hline S. prolifer Schwägr. & $\mathrm{C}$ & $\begin{array}{l}\text { AL, AM, BA, CE, DF, ES, } \\
\text { GO, MG, MT, PA, PE, PI, PR, } \\
\text { RJ, RO, RS, SC, SE, SP e TO }\end{array}$ & S.M.C. Siqueira 67 & HUVA \\
\hline \multicolumn{5}{|l|}{ Cryphaeaceae (1/1) } \\
\hline $\begin{array}{l}\text { Schoenobryum concavifolium (Griff.) } \\
\text { Gangulee }\end{array}$ & $\mathrm{C}$ & $\begin{array}{l}\text { BA, CE, DF, ES, GO, MG, PE, } \\
\text { PR, RJ, RO, RS, SC e SP }\end{array}$ & S.M.C. Siqueira 6, 9 & HUVA \\
\hline \multicolumn{5}{|l|}{ Dicranaceae (1/1) } \\
\hline Leucoloma tortellum (Mitt.) A. Jaeger & $\mathrm{C}$ & $\begin{array}{l}\text { AM, CE, MG, PA, RO, RR e } \\
\text { SP. }\end{array}$ & E.B. Souza 1808 & HUVA \\
\hline \multicolumn{5}{|l|}{ Fabroniaceae (1/1) } \\
\hline Fabronia ciliaris (Brid.) Brid. & $\mathrm{C}$ & $\begin{array}{l}\text { BA, CE, ES, GO, MG, PE, } \\
\text { PR, RJ, RS, SC e SP }\end{array}$ & $\begin{array}{l}\text { H.C. Oliveira 105, 162, } \\
167\end{array}$ & HUVA \\
\hline \multicolumn{5}{|l|}{ Fissidentaceae (1/12) } \\
\hline Fissidens angustifolius Sull. & $\mathrm{T}$ & $\begin{array}{l}\text { AC, AM, BA, CE, GO, PA, } \\
\text { RJ, RO e SP }\end{array}$ & S.M.C. Siqueira 52, 94 & HUVA \\
\hline F. asplenioides Hedw. & $\mathrm{R}$ & $\begin{array}{l}\text { BA, CE, ES, MG, MT, PR, RJ, } \\
\text { RS, RR, SC e SP }\end{array}$ & H.C. Oliveira 443 & HUEFS \\
\hline F. cryptoneuron P. de la Varde & $\mathrm{C}$ & CE e MG & H.C. Oliveira 161 & HUVA \\
\hline F. guianensis Mont. & $\mathrm{R} / \mathrm{T}$ & $\begin{array}{l}\text { AC, AL, AM, AP, BA, CE, } \\
\text { DF, ES, GO, MA, MG, MT, } \\
\text { MS, PA, PE, PI, RJ, RO, SC, } \\
\text { SP e TO }\end{array}$ & E.B. Souza 1907, 1908 & HUVA \\
\hline F. hornschuchii Mont. & $\mathrm{T}$ & $\begin{array}{l}\text { AL, AM, BA, CE, DF, ES, } \\
\text { GO, MA, MG, MS, MT, PA, } \\
\text { PE, PI, PR, RJ, RO, RS, SC e } \\
\text { SP }\end{array}$ & $\begin{array}{l}\text { E.B. Souza } 1905 \\
\text { M.D. Silva } 93\end{array}$ & HUVA \\
\hline
\end{tabular}


Tabela 1 (continuação)

\begin{tabular}{|c|c|c|c|c|}
\hline Filo/Família/Espécie & $\begin{array}{c}\text { Tipo de } \\
\text { substrato }\end{array}$ & $\begin{array}{c}\text { Distribuição geográfica } \\
\text { no Brasil }\end{array}$ & Voucher & Herbário \\
\hline F. intromarginatus (Hampe) A. Jaeger & $\mathrm{C}$ & $\begin{array}{l}\text { AC, BA, CE, ES, GO, MG, } \\
\text { MS, MT, PA, PR, RJ, RO, RS, } \\
\text { SC e SP }\end{array}$ & H.C. Oliveira 4, 181, 331 & HUVA \\
\hline F. minutus Thwaites \& Mitt. & $\mathrm{R}$ & CE, GO, MT, PA e SP & H.C. Oliveira 122 & HUVA \\
\hline F. prionodes Mont. & $\mathrm{R} / \mathrm{T}$ & $\begin{array}{l}\text { AC, AM, BA, CE, ES, GO, } \\
\text { MG, MT, PA, PB, PE, PI, PR, } \\
\text { RJ, RO, RR, RS e SP }\end{array}$ & $\begin{array}{l}\text { H.C. Oliveira 440, } 441 \text {, } \\
444,445\end{array}$ & HUVA \\
\hline F. radicans Mont. & $\mathrm{C}$ & $\begin{array}{l}\text { BA, CE, ES, MS, PA, PB, PE, } \\
\text { PI, PR, RJ, RS, SE e SP }\end{array}$ & M.D. Silva 38 & HUVA \\
\hline F. serratus Müll. Hal. & $\mathrm{T}$ & $\begin{array}{l}\text { AL, BA, CE, DF, GO, MG, } \\
\text { MT, PE, PI, PR, RJ, RS, SC, } \\
\text { SE e SP }\end{array}$ & H.C. Oliveira 163 & HUVA \\
\hline F. submarginatus Bruch & $\mathrm{C} / \mathrm{R}$ & $\begin{array}{l}\text { AC, AM, BA, CE, DF, ES, } \\
\text { GO, MA, MG, MS, MT, PA, } \\
\text { PB, PE, PI, RJ, RN, RO, RS, } \\
\text { SC, SP e TO }\end{array}$ & M.D. Silva 94, 96 & HUVA \\
\hline F. zollingeri Mont. & $\mathrm{T}$ & $\mathrm{AC}, \mathrm{AL}, \mathrm{AM}, \mathrm{AP}, \mathrm{BA}, \mathrm{CE}$ & H.C. Oliveira 201 & HUVA \\
\hline & & $\begin{array}{l}\text { DF, ES, GO, MA, MG, MS, } \\
\text { MT, PA, PB, PE, PI, PR, RJ, } \\
\text { RO, RR, RS, SC, SP e TO }\end{array}$ & & \\
\hline \multicolumn{5}{|l|}{ Leucobryaceae (3/3) } \\
\hline $\begin{array}{l}\text { Campylopus heterostachys (Hampe) } \\
\text { A. Jaeger }\end{array}$ & $\mathrm{C}$ & $\begin{array}{l}\text { BA, CE, GO, MG, MT, PI, } \\
\text { PR, RJ, RR, RS e SP }\end{array}$ & H.C. Oliveira 316 & HUVA \\
\hline Leucobryum crispum Müll. Hal. & $\mathrm{R} / \mathrm{T}$ & $\begin{array}{l}\text { AM, AP, BA, CE, DF, ES, GO, } \\
\text { MG, MT, PA, PR, RJ, RR, RS, } \\
\text { SC e SP }\end{array}$ & $\begin{array}{l}\text { H.C. Oliveira } 105 \\
\text { S.M.C. Siqueira } 90\end{array}$ & HUVA \\
\hline $\begin{array}{l}\text { Ochrobryum gardneri (Müll. Hal.) } \\
\text { Mitt. }\end{array}$ & $\mathrm{C}$ & $\begin{array}{l}\text { AL, AM, BA, CE, DF, ES, } \\
\text { GO, MG, MS, MT, PA, PE, } \\
\text { PR, RJ, RO, RR, SP e TO }\end{array}$ & H.C. Oliveira 344 & HUVA \\
\hline \multicolumn{5}{|l|}{ Orthotrichaceae $(2 / 3)$} \\
\hline $\begin{array}{l}{ }^{*} \text { Groutiella apiculata (Hook.) H.A. } \\
\text { Crum \& Steere }\end{array}$ & $\mathrm{C}$ & $\begin{array}{l}\text { BA, ES, MS, MT, PA, PE, PR, } \\
\text { RJ, SC e SP }\end{array}$ & E.B. Souza 1911 & HUVA \\
\hline $\begin{array}{l}\text { G. tomentosa (Hornsch.) Wijk \& } \\
\text { Margad. }\end{array}$ & $\mathrm{C} / \mathrm{R}$ & $\begin{array}{l}\text { AP, AM, BA, CE, MT, PA, } \\
\text { PE, RJ, RO, RS, SE e SP }\end{array}$ & S.M.C. Siqueira 2, 36 & HUVA \\
\hline $\begin{array}{l}\text { Macromitrium punctatum (Hook. \& } \\
\text { Grev.) Brid. }\end{array}$ & $\mathrm{R}$ & $\begin{array}{l}\text { AM, BA, CE, ES, GO, MG, } \\
\text { RJ e RS }\end{array}$ & H.C. Oliveira 191 & HUVA \\
\hline \multicolumn{5}{|l|}{ Pilotrichaceae (1/1) } \\
\hline $\begin{array}{l}\text { Lepidopilum cubense (Sull.) Mitt. } \\
\text { Pottiaceae (3/3) }\end{array}$ & EX & $\mathrm{BA}$ e $\mathrm{CE}$ & H.C. Oliveira 207 & HUVA \\
\hline Hyophila involuta (Hook.) A. Jaeger & $\mathrm{R}$ & $\begin{array}{l}\text { AM, BA, CE, DF, ES, GO, } \\
\text { MS, MT, PA, PB, PE, PI, PR, } \\
\text { RJ, RO, RS e SP }\end{array}$ & H.C. Oliveira 62, 197 & HUVA \\
\hline
\end{tabular}


Tabela 1 (continuação)

\begin{tabular}{|c|c|c|c|c|}
\hline Filo/Família/Espécie & $\begin{array}{c}\text { Tipo de } \\
\text { substrato }\end{array}$ & $\begin{array}{l}\text { Distribuição geográfica no } \\
\text { Brasil }\end{array}$ & Voucher & Herbário \\
\hline \multirow[t]{2}{*}{$\begin{array}{l}\text { Hyophiladelphus agrarius (Hedw.) } \\
\text { R.H. Zander }\end{array}$} & $\mathrm{R} / \mathrm{T}$ & $\mathrm{AC}, \mathrm{AM}, \mathrm{BA}, \mathrm{CE}, \mathrm{DF}, \mathrm{MG}$ & H.C. Oliveira 192, 196, & HUVA \\
\hline & & $\begin{array}{l}\text { MT, PA, PB, PE, RJ, RO, SE, } \\
\text { SP e TO }\end{array}$ & 313 & \\
\hline $\begin{array}{l}\text { Splachnobryum obtusum (Brid.) Müll. } \\
\text { Hal. }\end{array}$ & $\mathrm{T}$ & $\begin{array}{l}\text { AC, AL, AM, AP, CE, GO, } \\
\text { MS, PA, RS e SP }\end{array}$ & S.M.C. Siqueira 15 & HUVA \\
\hline \multicolumn{5}{|l|}{ Pterobryaceae $(3 / 5)$} \\
\hline $\begin{array}{l}\text { Henicodium geniculatum (Mitt.) } \\
\text { W.R. Buck }\end{array}$ & $\mathrm{C}$ & $\begin{array}{l}\text { AC, AL, AM, AP, BA, CE, } \\
\text { DF, ES, GO, MT, PA, PE, RJ, } \\
\text { RO, RS e SP }\end{array}$ & $\begin{array}{l}\text { H.C. Oliveira 237, 247, } \\
249,276,292\end{array}$ & HUVA \\
\hline Jaegerina scariosa (Lor.) Arzeni & $\mathrm{C}$ & $\begin{array}{l}\text { AL, AM, CE, DF, ES, GO, } \\
\text { MG, MS, MT, PA, PE, RJ, RR, } \\
\text { SC, SP e TO }\end{array}$ & S.M.C. Siqueira 93 & HUVA \\
\hline $\begin{array}{l}\text { * Orthostichopsis praetermissa W.R. } \\
\text { Buck }\end{array}$ & $\mathrm{C}$ & $\mathrm{AM}$ e BA & $\begin{array}{l}\text { S.M.C. Siqueira 27, 28, } \\
31,33,173,181\end{array}$ & HUVA \\
\hline O. tetragona (Sw. ex Hedw.) Broth. & $\mathrm{C}$ & AP, BA, CE, MG, PA, RS e SE & H.C. Oliveira 16 & HUVA \\
\hline * O. tortipilis (Müll. Hal.) Broth. & $\mathrm{C}$ & $\begin{array}{l}\text { AL, AM, BA, ES, MG, PE, } \\
\text { PR, RJ, SC e SP }\end{array}$ & S.M.C. Siqueira 172 & HUVA \\
\hline \multicolumn{5}{|l|}{ Pylaisiadelphaceae $(2 / 3)$} \\
\hline Isopterygium tenerifolium Mitt. & EX & $\begin{array}{l}\text { AM, BA, CE, DF, ES, GO, } \\
\text { MG, MT, PA, PR, RJ, RO, } \\
\text { RR, RS, SC, SP e TO }\end{array}$ & S.M.C. Siqueira 174 & HUVA \\
\hline I. tenerum (Sw.) Mitt. & C/EX & $\begin{array}{l}\text { AC, AM, AP, BA, CE, DF, ES, } \\
\text { GO, MG, MS, MT, PA, PB, } \\
\text { PE, PI, PR, RJ, RO, RR, RS, } \\
\text { SC, SP e TO }\end{array}$ & S.M.C. Siqueira 72, 158 & HUVA \\
\hline $\begin{array}{l}\text { Pterogonidium pulchellum (Hook.) } \\
\text { Müll. Hal. }\end{array}$ & C/EX & $\begin{array}{l}\text { AM, AP, BA, CE, PA, PE, RJ, } \\
\text { RO e SP }\end{array}$ & $\begin{array}{l}\text { H.C. Oliveira 177, } 178 \text {, } \\
179\end{array}$ & HUVA \\
\hline \multicolumn{5}{|l|}{ Racopilaceae (1/1) } \\
\hline $\begin{array}{l}\text { Racopilum tomentosum (Sw. ex } \\
\text { Hedw.) Brid. }\end{array}$ & $\mathrm{C} / \mathrm{R}$ & $\begin{array}{l}\text { AC, AL, AM, BA, CE, DF, ES, } \\
\text { GO, MG, MS, MT, PA, PE, } \\
\text { PR, RJ, RO, RS, SC e SP }\end{array}$ & $\begin{array}{l}\text { H.C. Oliveira 2, } 25 \\
\text { S.M.C. Siqueira 17, } 60\end{array}$ & HUVA \\
\hline \multicolumn{5}{|l|}{ Sematophyllaceae $(2 / 6)$} \\
\hline $\begin{array}{l}\text { Sematophyllum adnatum (Michx.) } \\
\text { E. Britton }\end{array}$ & $\mathrm{C}$ & $\begin{array}{l}\text { AM, BA, DF, ES, GO, MT, } \\
\text { PA, PE, RJ, RS, SC, SP e TO }\end{array}$ & P.S. Costa 5 & HUVA \\
\hline S. cuspidiferum Mitt. & $\mathrm{C}$ & CE, GO, MG, MS, MT e SP & P.S. Costa 17 & HUVA \\
\hline S. galipense (Müll. Hal.) Mitt. & $\mathrm{C}$ & $\begin{array}{l}\text { BA, ES, GO, MG, MT, PA, } \\
\text { PE, PR, RJ, RO, RR, RS, SC, } \\
\text { SP e TO }\end{array}$ & S.M.C. Siqueira 7, 15, 30 & HUVA \\
\hline S. subpinnatum (Brid.) E. Britton & $\mathrm{C}$ & $\begin{array}{l}\text { AC, AL, AM, AP, BA, CE, } \\
\text { DF, ES, GO, MG, MS, MT, } \\
\text { PA, PB, PE, PR, RJ, RO, RR, } \\
\text { RS, SC, SP e TO }\end{array}$ & $\begin{array}{l}\text { H.C. Oliveira 173, 174, } \\
200,210,221,233,234, \\
239\end{array}$ & HUVA \\
\hline
\end{tabular}


Tabela 1 (continuação)

\begin{tabular}{|c|c|c|c|c|}
\hline Filo/Família/Espécie & $\begin{array}{l}\text { Tipo de } \\
\text { substrato }\end{array}$ & $\begin{array}{c}\text { Distribuição geográfica } \\
\text { no Brasil }\end{array}$ & Voucher & Herbário \\
\hline S. subsimplex (Hedw.) Mitt. & $\mathrm{C} / \mathrm{R}$ & $\begin{array}{l}\text { AC, AM, AP, BA, CE, DF, ES, } \\
\text { GO, MA, MG, MS, MT, PA, } \\
\text { PB, PE, PI, PR, RJ, RO, RR, } \\
\text { RS, SC, SE, SP e TO }\end{array}$ & H.C. Oliveira 90, 93, 119 & HUVA \\
\hline $\begin{array}{l}\text { Trichosteleum intricatum (Thér.) J. } \\
\text { Florsch }\end{array}$ & $\mathrm{EF}$ & CE, DF e PA & H.C. Oliveira 182 & HUVA \\
\hline \multicolumn{5}{|l|}{ Stereophyllaceae (1/1) } \\
\hline $\begin{array}{l}\text { Eulacophyllum cultelliforme (Sull.) } \\
\text { W.R. Buck \& Ireland }\end{array}$ & $\mathrm{C} / \mathrm{R}$ & $\begin{array}{l}\text { AM, BA, CE, ES, MG, MS, } \\
\text { MT, PB, PE, PR, RJ, RS, SE, } \\
\text { SP e TO }\end{array}$ & H.C. Oliveira 121, 324 & HUVA \\
\hline \multicolumn{5}{|l|}{ MARCHANTIOPHYTA (10/22/42) } \\
\hline \multicolumn{5}{|l|}{ Calypogeiaceae (1/1) } \\
\hline Calypogeia laxa Gottsche \& Lindenb. & $\mathrm{EX} / \mathrm{T}$ & $\begin{array}{l}\text { AM, CE, DF, ES, MG, PA, } \\
\text { PE, RJ, SC e SP }\end{array}$ & $\begin{array}{l}\text { H.C. Oliveira 103, 109, } \\
118,131,440\end{array}$ & $\begin{array}{l}\text { HUVA } \\
\text { HUEFS }\end{array}$ \\
\hline \multicolumn{5}{|l|}{ Cephaloziellaceae (1/1) } \\
\hline $\begin{array}{l}\text { Cylindrocolea planifolia (Steph.) R.M. } \\
\text { Schust. }\end{array}$ & $\mathrm{R}$ & BA, CE, MG, RJ, RO, SC e SP & S.M.C. Siqueira 100 & HUVA \\
\hline \multicolumn{5}{|l|}{ Fossombroniaceae (1/1) } \\
\hline $\begin{array}{l}\text { Fossombronia porphyrorhiza (Nees) } \\
\text { Prosk. }\end{array}$ & $\mathrm{R}$ & $\begin{array}{l}\text { BA, CE, DF, ES, GO, MG, } \\
\text { MS, MT, PE, RJ, SP e TO }\end{array}$ & $\begin{array}{l}\text { H.C. Oliveira } 81,83,86 \text {, } \\
145\end{array}$ & HUVA \\
\hline \multicolumn{5}{|l|}{ Frullaniaceae $(1 / 3)$} \\
\hline Frullania caulisequa (Nees) Nees & EX & $\begin{array}{l}\text { AC, AL, BA, CE, DF, ES, GO, } \\
\text { MG, MT, PA, PE, RJ, RR, RS, } \\
\text { SC, SE e SP }\end{array}$ & H.C. Oliveira 425 & HUEFS \\
\hline F. dusenii Steph. & $\mathrm{R}$ & $\begin{array}{l}\text { AL, CE, ES, GO, MG, PE, RJ, } \\
\text { RR, RS, SC, SE e SP }\end{array}$ & M.D. Silva 61 & HUVA \\
\hline F. riojaneirensis (Raddi) Spruce & EX & $\begin{array}{l}\text { AL, BA, CE, DF, ES, GO, } \\
\text { MG, MS, MT, PA, PB, PE, } \\
\text { PR, RJ, RS, SC, SE e SP }\end{array}$ & S.M.C. Siqueira 38, 49 & HUVA \\
\hline \multicolumn{5}{|l|}{ Lejeuneaceae $(12 / 23)$} \\
\hline $\begin{array}{l}\text { Acrolejeunea torulosa (Lehm. \& } \\
\text { Lindenb.) Schiffn. }\end{array}$ & $\mathrm{C}$ & $\begin{array}{l}\text { AC, AL, AM, BA, CE, ES, } \\
\text { GO, MA, MG, MS, MT, PA, } \\
\text { PR, RJ, RO, RR, SP e TO }\end{array}$ & S.M.C. Siqueira 48 & HUVA \\
\hline $\begin{array}{l}\text { Aphanolejeunea camillii (Lehm.) R.M. } \\
\text { Schust. }\end{array}$ & $\mathrm{EF}$ & $\begin{array}{l}\text { AL, AM, CE, MG, PA, RJ e } \\
\text { SP. }\end{array}$ & E.B. Souza 2262 & HUVA \\
\hline${ }^{*}$ A. kunertiana Steph. & $\mathrm{EF}$ & BA, MG, RS e SP & S.M.C. Siqueira 77 & HUVA \\
\hline Bryopteris diffusa (Sw.) Nees & $\mathrm{C} / \mathrm{EX}$ & $\begin{array}{l}\text { AC, AL, AM, BA, CE, ES, } \\
\text { MG, MT, PA, PE, PR, RJ, RS, } \\
\text { SC, SE e SP }\end{array}$ & M.D. Silva 22, 55, 57 & HUVA \\
\hline $\begin{array}{l}\text { Cheilolejeunea clausa (Nees \& Mont.) } \\
\text { R.M. Schust. }\end{array}$ & $\mathrm{C} / \mathrm{EX}$ & $\mathrm{AC}, \mathrm{AL}, \mathrm{AM}, \mathrm{AP}, \mathrm{BA}, \mathrm{CE}$ & H.C. Oliveira 429, 431 & HUEFS \\
\hline & & $\begin{array}{l}\text { ES, GO, MG, MS, MT, PA, } \\
\text { PE, PR, RJ, RS, SC e SP }\end{array}$ & & \\
\hline
\end{tabular}


Tabela 1 (continuação)

\begin{tabular}{|c|c|c|c|c|}
\hline Filo/Família/Espécie & $\begin{array}{c}\text { Tipo de } \\
\text { substrato }\end{array}$ & $\begin{array}{c}\text { Distribuição geográfica } \\
\text { no Brasil }\end{array}$ & Voucher & Herbário \\
\hline $\begin{array}{l}\text { C. discoidea (Lehm. \& Lindenb.) } \\
\text { Kachroo \& R.M. Schust. }\end{array}$ & $\mathrm{C} / \mathrm{R}$ & $\begin{array}{l}\text { AL, BA, CE, DF, ES, GO, } \\
\text { MG, MS, MT, RJ, RS, SE e SP }\end{array}$ & $\begin{array}{l}\text { E.B. Souza } 1811 \\
\text { H.C. Oliveira } 425\end{array}$ & HUEFS \\
\hline C. rigidula (Mont.) R.M. Schust. & $\mathrm{C}$ & $\begin{array}{l}\text { AC, AL, AM, AP, BA, CE, } \\
\text { DF, ES, GO, MG, MS, MT, } \\
\text { PA, PB, PE, PR, RJ, RR, RS, } \\
\text { SC, SE, SP e TO }\end{array}$ & H.C. Oliveira 426, 439 & HUEFS \\
\hline C. unciloba (Lindenb.) Malombe & EX & $\begin{array}{l}\text { AC, AM, BA, CE, ES, MG, } \\
\text { PA, PE, RJ, RS, SC e SP }\end{array}$ & H.C. Oliveira 103 & HUVA \\
\hline $\begin{array}{l}\text { C. xanthocarpa (Lehm. \& Lindenb.) } \\
\text { Malombe }\end{array}$ & $\mathrm{C} / \mathrm{EX}$ & $\begin{array}{l}\text { AL, BA, CE, ES, GO, MG, } \\
\text { PE, RJ, RS, SC e SP }\end{array}$ & $\begin{array}{l}\text { S.M.C. Siqueira 160, 167, } \\
\text { 171, } 179\end{array}$ & HUVA \\
\hline Cololejeunea subcardiocarpa Tixier & $\mathrm{EF}$ & $\begin{array}{l}\text { AC, AM, BA, CE, ES, GO, } \\
\text { MG, MT, PA, PE, PR, RJ e SP }\end{array}$ & E.B. Souza 2263 & HUVA \\
\hline Diplasiolejeunea cavifolia Steph. & EX & $\begin{array}{l}\text { AL, BA, CE, PE, PR, RJ, SC } \\
\text { e SP }\end{array}$ & H.C. Oliveira 425 & HUEFS \\
\hline $\begin{array}{l}\text { Drepanolejeunea anoplantha (Spruce) } \\
\text { Steph. }\end{array}$ & $\mathrm{C}$ & AM, BA, CE, ES, RJ e SP & H.C. Oliveira 20 & HUVA \\
\hline D. fragilis Bischl. & $\mathrm{EX} / \mathrm{EF}$ & $\begin{array}{l}\text { AL, AM, AP, BA, CE, MG, } \\
\text { PA, PE, RJ, RR, SE e SP }\end{array}$ & H.C. Oliveira 425, 437 & HUEFS \\
\hline Lejeunea capensis Gottsche & $\mathrm{C}$ & CE, MG, PR, RJ, RS e SP & H.C. Oliveira 132 & HUVA \\
\hline $\begin{array}{l}\text { L. caulicalyx (Steph.) E. Reiner \& } \\
\text { Goda }\end{array}$ & $\mathrm{C}$ & $\begin{array}{l}\text { AC, AL, BA, CE, ES, MS, } \\
\text { MT, PE, PR, RJ, RR e SP }\end{array}$ & H.C. Oliveira 17, 63 & HUVA \\
\hline L. flava (Sw.) Nees & $\mathrm{C} / \mathrm{EF} / \mathrm{EX}$ & $\begin{array}{l}\text { AC, AL, AM, BA, CE, DF, } \\
\text { ES, GO, MG, MS, MT, PA, } \\
\text { PB, PE, PR, RJ, RR, RS, SC, } \\
\text { SE e SP }\end{array}$ & $\begin{array}{l}\text { H.C. Oliveira } 38,134 \\
\text { S.M.C. Siqueira } 62,100 \text {, } \\
162\end{array}$ & HUVA \\
\hline L. filipes Spruce & $\mathrm{C}$ & BA, CE, ES, MS, MT e SP & S.M.C. Siqueira 170 & HUVA \\
\hline L. immersa Spruce & $\mathrm{C}$ & $\begin{array}{l}\text { AL, AM, BA, CE, PE, RJ, RO } \\
\text { e SP }\end{array}$ & H.C. Oliveira 431 & HUEFS \\
\hline L. laetevirens Nees \& Mont. & $\mathrm{C}$ & $\begin{array}{l}\text { AC, AL, AM, AP, BA, CE, } \\
\text { DF, ES, GO, MG, MS, MT, } \\
\text { PA, PB, PE, RJ, RR, RS, SC, } \\
\text { SE e SP }\end{array}$ & $\begin{array}{l}\text { H.C. Oliveira 138, 158, } \\
220\end{array}$ & HUVA \\
\hline $\begin{array}{l}\text { Leptolejeunea elliptica (Lehm. \& } \\
\text { Lindenb.) Schiffn. }\end{array}$ & $\mathrm{EF}$ & $\begin{array}{l}\text { AC, AL, AM, AP, BA, CE, } \\
\text { DF, ES, GO, MG, MT, PA, } \\
\text { PE, PR, RJ, RR, SC, SE e SP }\end{array}$ & H.C. Oliveira 437, 438 & HUEFS \\
\hline Marchesinia brachiata (Sw.) Schiffn. & $\mathrm{C}$ & $\begin{array}{l}\text { BA, CE, ES, MG, PE, PR, RJ, } \\
\text { RR, SC, SE e SP }\end{array}$ & M.D. Silva 54, 60, 87 & HUVA \\
\hline Microlejeunea epiphylla Bischl. & EX & $\begin{array}{l}\text { AL, AP, BA, CE, ES, GO, } \\
\text { MG, MS, PA, PB, PE, RS, SE, } \\
\text { SP e TO }\end{array}$ & H.C. Oliveira 425 & HUEFS \\
\hline $\begin{array}{l}\text { Odontolejeunea lunulata (F. Weber) } \\
\text { Schiffner }\end{array}$ & $\mathrm{EF}$ & $\begin{array}{l}\text { AC, AL, AM, AP, BA, CE, ES, } \\
\text { MG, MT, PA, PE, PR, RJ, RR, } \\
\text { RS e SP }\end{array}$ & $\begin{array}{l}\text { S.M.C. Siqueira 79, 164, } \\
166\end{array}$ & HUVA \\
\hline
\end{tabular}

Lophocoleaceae $(1 / 2)$ 
Tabela 1 (continuação)

\begin{tabular}{|c|c|c|c|c|}
\hline Filo/Família/Espécie & $\begin{array}{c}\text { Tipo de } \\
\text { substrato }\end{array}$ & $\begin{array}{c}\text { Distribuição geográfica } \\
\text { no Brasil }\end{array}$ & Voucher & Herbário \\
\hline Lophocolea bidentata (L.) Dumort. & $\mathrm{R}$ & $\begin{array}{l}\text { AC, AM, BA, CE, DF, ES, } \\
\text { GO, MG, MS, MT, PE, PR, } \\
\text { RJ, RR, RS, SC e SP }\end{array}$ & H.C. Oliveira 123 & HUVA \\
\hline${ }^{\star}$ L. liebmanniana Gottsche & $\mathrm{R}$ & $\begin{array}{l}\text { AC, AL, AM, AP, DF, GO, } \\
\text { MT, PA, PE e SP }\end{array}$ & P.S. Costa 46 & HUVA \\
\hline \multicolumn{5}{|l|}{ Metzgeriaceae (1/2) } \\
\hline Metzgeria aurantiaca Steph. & EX & $\begin{array}{l}\text { AM, BA, CE, ES, MG, PB, } \\
\text { PE, PR, RJ, RS, SC e SP }\end{array}$ & H.C. Oliveira 310, 311 & HUVA \\
\hline M. furcata (L.) Dumort. & $\mathrm{C}$ & $\begin{array}{l}\text { AC, BA, CE, ES, GO, MG, } \\
\text { PE, PR, RJ, RS, SC e SP }\end{array}$ & $\begin{array}{l}\text { H.C. Oliveira } 75,106 \text {, } \\
124,160,308\end{array}$ & HUVA \\
\hline \multicolumn{5}{|l|}{ Pallaviciniaceae $(2 / 2)$} \\
\hline Pallavicinia lyellii (Hook.) Gray & $\mathrm{R} / \mathrm{T}$ & $\begin{array}{l}\text { AC, AM, BA, CE, DF, GO, } \\
\text { MS, MT, PA, RJ, RS, SC, SP } \\
\text { e TO }\end{array}$ & H.C. Oliveira 107, 447 & $\begin{array}{l}\text { HUVA } \\
\text { HUEFS }\end{array}$ \\
\hline Symphyogyna aspera Steph. & $\mathrm{R}$ & $\begin{array}{l}\text { AM, BA, CE, DF, ES, GO, } \\
\text { MG, MS, MT, PA, PE, RJ, RS, } \\
\text { SC e SP }\end{array}$ & H.C. Oliveira 446 & HUEFS \\
\hline \multicolumn{5}{|l|}{ Plagiochilaceae $(1 / 6)$} \\
\hline $\begin{array}{l}\text { Plagiochila corrugata (Nees) Nees \& } \\
\text { Mont. }\end{array}$ & $\mathrm{C}$ & $\begin{array}{l}\text { AC, BA, CE, DF, ES, GO, } \\
\text { MG, PE, PR, RJ, RS, SC e SP }\end{array}$ & S.M.C. Siqueira 23 & HUVA \\
\hline $\begin{array}{l}\text { P. disticha (Lehm. \& Lindenb.) } \\
\text { Lindenb. }\end{array}$ & $\mathrm{C}$ & $\begin{array}{l}\text { AC, AL, AM, AP, BA, CE, } \\
\text { DF, ES, GO, MG, MS, MT, } \\
\text { PA, PB, PE, RJ, RR, RS, SC } \\
\text { e SP }\end{array}$ & H.C. Oliveira 31 & HUVA \\
\hline P. martiana (Nees) Lindenb. & $\mathrm{C}$ & $\begin{array}{l}\text { AC, AL, BA, CE, DF, ES, GO, } \\
\text { MG, MS, MT, PA, PE, PR, RJ, } \\
\text { RS, SC e SP }\end{array}$ & $\begin{array}{l}\text { H.C. Oliveira } 35,42,101 \text {, } \\
129,216,304\end{array}$ & HUVA \\
\hline P. micropteryx Gottsche & $\mathrm{R}$ & $\begin{array}{l}\text { AC, BA, CE, ES, MG, PA, RJ, } \\
\text { RS e SP }\end{array}$ & H.C. Oliveira 120 & HUVA \\
\hline P. patula (Sw.) Lindenb. & $\mathrm{C}$ & $\begin{array}{l}\text { AC, BA, CE, DF, MG, RJ, SC } \\
\text { e SP }\end{array}$ & H.C. Oliveira 439 & HUEFS \\
\hline P. raddiana Lindenb. & $\mathrm{C}$ & $\begin{array}{l}\text { AC, AM, BA, CE, ES, GO, } \\
\text { MG, MT, PA, PE, PR, RJ, RS, } \\
\text { SC e SP }\end{array}$ & H.C. Oliveira 428,430 & HUEFS \\
\hline \multicolumn{5}{|l|}{ Ricciaceae $(1 / 1)$} \\
\hline Riccia stenophylla Spruce & $\mathrm{C} / \mathrm{R}$ & $\begin{array}{l}\text { BA, CE, ES, GO, MG, MS, } \\
\text { MT, PE, PR, RJ, RS, SC e SP }\end{array}$ & H.C. Oliveira 10, 43 & HUVA \\
\hline
\end{tabular}

Quanto aos substratos colonizados, a preferência por apenas um tipo de substrato foi de $77 \%$, percentual elevado quando comparado a outros trabalhos como os de Visnadi (2005) e Santos \& Costa (2008). As briófitas corticícolas foram predominantes $(51 \%$ da amostragem), seguidas de rupícolas $(16 \%)$, terrícolas (14\%), epíxilas $(11 \%)$ e epífilas $(8 \%)$. A grande representatividade de plantas corticícolas deveu-se à alta disponibilidade de árvores e arbustos. Esse grupo foi bem representado principalmente por espécies da família Lejeuneaceae que, de acordo com Pócs (1982) e Richards (1984), se destacam em Florestas Tropicais, colonizando variados substratos, especialmente troncos vivos e mortos, bem como outros favoráveis ao estabelecimento de briófitas.

Em relação à distribuição geográfica das espécies 
no Brasil, foi constatado que $12 \%$ dos táxons apresentam distribuição restrita, entre eles: Philonotis longiseta (Michx.) E. Britton, Fissidens cryptoneuron P. de la Varde, Lepidopilum cubense (Sull.) Mitt. e Orthostichopsis praetermissa W.R. Buck. A maior parte das espécies apresenta ampla distribuição, com $54 \%$ ocorrendo em seis a quinze Estados e $34 \%$ em praticamente todo o país.

Os dados obtidos foram comparados a outros registros de briófitas para fragmentos de Mata Atlântica no Estado da Bahia (Valente \& Pôrto 2006, Valente et al. 2009) mostrando que $24 \%$ das hepáticas e $26 \%$ dos musgos são comuns com o presente estudo. Já para o Estado de Pernambuco essa correspondência chega a $22 \%$ das espécies (Costa et al. 2010). Considerando-se a listagem de briófitas para a Mata Atlântica (Costa et al. 2009a, b, c), evidencia-se uma correlação de $89 \%$ dos táxons.

Os resultados alcançados demonstram a significativa riqueza de briófitas do Sítio São Luís, apresentando $61 \%$ das espécies listadas para a Ibiapaba Norte e $36 \%$ das citações de briófitas para o Estado do Ceará. Além disso, enfatiza-se a necessidade de que novas coletas sejam realizadas nesse fragmento, visto que, esse espaço foi recentemente incorporado à área do Parque Nacional de Ubajara.

\section{Agradecimentos}

Os autores agradecem à Fundação Cearense de Apoio ao Desenvolvimento Científico e Tecnológico (FUNCAP), pelas bolsas de Iniciação Científica concedidas às duas primeiras autoras e pela bolsa de Produtividade concedida ao terceiro autor; à Universidade Estadual Vale do Acaraú (UVA), pelo apoio logístico; ao Dr. Cid José Passos Bastos, do Laboratório de Taxonomia de Briófitas da Universidade Federal da Bahia (UFBA), pelo auxílio na confirmação de algumas espécies do gênero Cheilolejeunea; ao Dr. Denilson Fernandes Peralta, do Instituto de Botânica de São Paulo (IBt), pelo envio de literatura e às biólogas Evelyne Marreira, Ellen Kallyne Brandão, Denismara Lima e Maria Cátia Barroso, pelo auxílio durante as coletas.

\section{Literatura citada}

Allen, B.H. 2002. Moss Flora of Central America. Part 2. Encalyptaceae-Orthotrichaceae. Monographs in Systematic Botany from the Missouri Botanical Garden 90: 1-699.

Bezerra, E.C., Bezerra, J.E.G. \& Mende, M.F.S. 1989. Precipitações. In: M.L.R. Martins (coord.). Atlas do Ceará. IPLANCE, Fortaleza, pp. 18-19.
Bischler, H. 1964. Le genre Drepanolejeunea Steph. in Amérique Centrale et Méridionale. Revue Bryologique et Lichénologique 33: 95-134.

Brito, A.E.R.M. \& Pôrto, K.C. 2000. Guia de Estudos de Briófitas: Briófitas do Ceará. Edições UFC, Fortaleza.

Buck, W.R. 1998. Pleurocarpous Mosses of the West Indies. Memoirs of The New York Botanical Garden 82: 1-400.

Churchill, S.P. 1988. A revision of the moss genus Lepidopilum (Callicostaceae). PhD Dissertation, City University of New York, New York.

Costa, D.P. 2009. Briófitas. In: J.R. Stehmann, R.C. Forzza, A. Salino, M. Cabral, D.P. Costa \& L.H.Y. Kamino (eds.). Plantas da Floresta Atlântica. Instituto de Pesquisas Jardim Botânico do Rio de Janeiro, Rio de Janeiro, pp.13-17.

Costa, D.P. 2010. Briófitas. In: R.C. Forzza, J.F.A. Baumgratz, C.E.M. Bicudo, A.A.A. Carvalho Jr., A. Costa, D.P. Costa, M. Hopkins, P.M. Leitman, L.G. Lohmann, L.C. Maia, G. Martinelli, M. Menezes, M.P. Morim, M.A.N. Coelho, A.L. Peixoto, J.R. Pirani, J. Prado, L.P. Queiroz, V.C. Souza, J.R. Stehmann, L.S. Sylvestre, B.M.T. Walter \& D. Zappi (eds.). Catálogo de Plantas e Fungos do Brasil. v.1. Andrea Jakobsson Estúdio \& Instituto de Pesquisas Jardim Botânico do Rio de Janeiro, Rio de Janeiro, pp. 452-521.

Costa, D.P. \& Santos, N.D. 2009. Conservação de hepáticas na Mata Atlântica do sudeste do Brasil: uma análise regional no Estado do Rio de Janeiro. Acta Botanica Brasilica 23: 913-922.

Costa, D.P. \& Silva, A.G. 2003. Briófitas da Reserva Natural da Vale do Rio Doce, Linhares, Espírito Santo, Brasil. Boletim do Museu de Biologia Mello Leitão 16: 21-38.

Costa, D.P., Imbassahy, C.A.A. \& Silva, V.P.A.V. 2005. Checklist and distribution of mosses, liverworts and hornworts of Rio de Janeiro State, Brazil. The Journal of the Hattori Botanical Laboratory 98: 259-298.

Costa, D.P., Santos, N.D., Imbassahy, C.A.A. \& Lopes, D.J. 2009a. Briófitas-Antóceros. In: J.R. Stehmann, R.C. Forzza, A. Salino, M. Cabral, D.P. Costa \& L.H.Y. Kamino (eds.). Plantas da Floresta Atlântica. Instituto de Pesquisas Jardim Botânico do Rio de Janeiro, Rio de Janeiro, pp.43.

Costa, D.P., Santos, N.D., Imbassahy, C.A.A. \& Lopes, D.J. 2009b. Briófitas-Hepáticas. In: J.R. Stehmann, R.C. Forzza, A. Salino, M. Cabral, D.P. Costa \& L.H.Y. Kamino (eds.). Plantas da Floresta Atlântica. Instituto de Pesquisas Jardim Botânico do Rio de Janeiro, Rio de Janeiro, pp. 43-56.

Costa, D.P., Porto, K.C., Luizi-Ponzo, A.P., Ilkiu-Borges, A.L., Bastos, C.J.P., Câmara, P.E.A.S., Bôas-Bastos, S.V., Vaz-Imbassahy, T.F., Imbassahy, C.A.A. \& Lopes, D.J. 2009c. Briófitas-Musgos. In: J.R. Stehmann, R.C. Forzza, A. Salino, M. Cabral, D.P. Costa \& L.H.Y. Kamino (eds.). Plantas da Floresta Atlântica. Instituto de Pesquisas Jardim Botânico do Rio de Janeiro, Rio de Janeiro, pp. 57-80. 
Crandall-Stottler, B., Stottler, R.E. \& Long, D.G. 2009. Phylogeny and classification of the Marchantiophyta. Edinburgh Journal of Botany 66: 155-198.

Fernandes, A. 1990. Temas fitogeográficos. Stylus comunicações, Fortaleza.

Figueiredo, M.A. 1989. Vegetação. In: M.L.R. Martins (coord.). Atlas do Ceará. IPLANCE, Fortaleza, pp. 24-25.

Florschütz, P.A. 1964. The mosses of Suriname. Part. I. Flora Suriname 6: 1-271.

Fonseca, G.A.B. 1985. The vanishing Brazilian Atlantic Forest. Biological Conservation 34: 17-34.

Galindo-Leal, C. \& Câmara, I.G. 2005. Status do hotspot Mata Atlântica: uma síntese. In: C. Galindo-Leal \& I.G. Câmara (eds.). Mata Atlântica: biodiversidade, ameaças e perspectivas. Fundação SOS Mata AtlânticaConservação Internacional, Belo Horizonte, pp. 3-12.

Germano, S.R. \& Pôrto, K.C. 1996. Floristic survey of epixylic bryophytes of an area remnant of the Atlantic Forest (Timbaúba-PE, Brazil). Tropical Bryology 12: 21-28.

Germano, S.R. \& Pôrto, K.C. 1998. Briófitas epíxilas de uma área remanescente de Floresta Atlântica (Timbaúba, PE, Brasil). 2. Lejeuneaceae. Acta Botanica Brasilica 12: 53-66.

Goffinet, B., Buck, W.R. \& Shaw, A.J. 2008. Morphology and classification of the Bryophyta. In: B. Goffinet \& A.J. Shaw (eds.). Bryophyte Biology. Cambridge University Press, Cambridge, pp. 55-138.

Gradstein, S.R. 1994. Lejeuneaceae: Ptychantheae, Brachiolejeuneae. Flora Neotropica Monograph 62: 1-216.

Gradstein, S.R. \& Costa, D.P. 2003. The Hepaticae and Anthocerotae of Brazil. Memoirs of The New York Botanical Garden 87: 1-318.

Gradstein, S.R., Churchill, S.P. \& Salazar-Allen, N. 2001. Guide to the bryophytes of Tropical America. Memoirs of the New York Botanical Garden 86: 1-577.

Hässel-de-Menéndez, G.G. 1962. Estudio de las Anthocerotales y Marchantiales de la Argentina. Opera Lilloana 7: 1-297.

Hell, K.G. 1969. Briófitas talosas dos arredores da cidade de São Paulo (Brasil). Boletim de Botânica da Faculdade de Filosofia, Ciências e Letras da Universidade de São Paulo, Botânica 25: 1-187.

Heinrichs, J. \& Gradstein, S.R. 2000. A revision of Plagiochila sect. Crispatae and sect. Hypnoides (Hepaticae) in the Neotropics. I. Plagiochila disticha, $P$. montagnei and P. raddiana. Nova Hedwigia 70: 161-184.

Heinrichs, J., Gradstein, S.R. \& Grolle, R. 1998. A revision of the neotropical species of Plagiochila (Dumort.) Dumort. (Hepaticae) described by Olof Swartz. The Journal of the Hattori Botanical Laboratory 85: 1-32.
Ilkiu-Borges, A.L. \& Lisboa, R.C.L. 2002. Os gêneros Lejeunea e Microlejeunea (Lejeuneaceae) na estação Científica Ferreira Penna, Estado do Pará, Brasil, e novas ocorrências. Acta Amazonica 32: 541-553.

Inoue, H. 1989. Notes on the Plagiochilaceae, XVI. Studies on some Plagiochila species in the Neotropics. Bulletin of the Natural Science Museum, Series B 15: 35-47.

Leite, F.A.B. \& Marques, J.N. 1989. Solos. In: M.L.R. Martins (coord.). Atlas do Ceará. IPLANCE, Fortaleza, pp. 16-17.

Lemos-Michel, E. \& Yano, O. 1998. O gênero Bryopteris (Hepatophyta) no Brasil. Acta Botanica Brasilica 12: 5-24.

Mori, S.A., Boom, B.M. \& Prance, G.T. 1981. Distribution patterns and conservation of eastern Brazilian coastal forest tree species. Brittonia 33: 233-245.

Oliveira-e-Silva, M.I.M.N. \& Yano, O. 2000. Anthocerotophyta e Hepatophyta de Mangaratiba e Angra dos Reis, Rio de Janeiro, Brasil. Boletim do Instituto de Botânica 13: 1-102.

Oliveira-e-Silva, M.I.M.N., Milanez, A.I. \& Yano, O. 2002. Aspectos ecológicos de briófitas em áreas preservadas de Mata Atlântica, Rio Janeiro, Brasil. Tropical Bryology 22: 77-102.

Oliveira, H.C. \& Alves, M.H. 2007. Adições à brioflora do Estado do Ceará, Brasil. Rodriguésia 58: 1-11.

Oliveira, H.C. \& Bastos, C.J.P. 2009a. Jungermanniales (Marchantiophyta) da Chapada da Ibiapaba, Ceará, Brasil. Acta Botanica Brasilica 23: 1202-1209.

Oliveira, H.C. \& Bastos, C.J.P. 2009b. Antóceros (Anthocerotophyta) e hepáticas talosas (Marchantiophyta) da Chapada da Ibiapaba, Ceará, Brasil. Rodriguésia 60: 477-484.

Oliveira, H.C. \& Bastos, C.J.P. 2010a. Musgos pleurocárpicos da Chapada da Ibiapaba, Ceará, Brasil. Acta Botanica Brasilica 24: 193-204.

Oliveira, H.C. \& Bastos, C.J.P. 2010b. Fissidentaceae (Bryophyta) da Chapada da Ibiapaba, Ceará, Brasil. Revista Brasileira de Botânica 33: 393-405.

Peralta, D.F. \& Athayde Filho, F.P. 2008. Briófitas corticícolas de mata ciliar ao longo do Rio Uruguai, antes do alagamento da área pela Barragem de Itá, entre Santa Catarina e Rio Grande do Sul, Brasil. Hoehnea 35: 411-418.

Pereira, M.S. \& Alves, R.R.N. 2006. Composição florística de um remanescente de Mata Atlântica na Área de Proteção Ambiental Barra do Rio Mamanguape, Paraíba, Brasil. Revista de Biologia e Ciências da Terra 6: 357-366.

Pócs, T. 1982. The Tropical Bryophytes. In: A.J.E. Smith (ed.). Bryophyte Ecology. Chapman \& Hall, London, pp. 54-104.

Pursell, R.A. 2008. Fissidentaceae. Flora Neutrotropica Monograph 101: 1-278. 
Reiner-Drehwald, M.E. 2000. Las Lejeuneaceae (Hepaticae) des Misiones, Argentina VI. Lejeunea y Taxilejeunea. Tropical Bryology 19: 81-131.

Renzaglia, K.S., Villarreal, J.C. \& Duff, R.J. 2008. New insights into morphology, anatomy, and systematics or hornworts. In: B. Goffinet \& A.J. Shaw (eds.). Bryophyte Biology. Cambridge University Press, Cambridge, pp. 139-171.

Ribeiro, M.C., Metzger, J.P., Martensen, A.C., Ponzoni, F.J. \& Hirota, M.M. 2009. The Brazilian Atlantic Forest: How much is left, and how is the remaining forest distributed? Implications for conservation. Biological Conservation 142: 1144-1156.

Richards, P.W. 1984. The ecology of tropical forest bryophytes. In: R.M. Schuster (ed.). New Manual of Bryology. The Hattori Botanical Laboratory, Nichinan, pp. 1233-1270.

Robbins, R.G. 1952. Bryophyte ecology of a dune area in New Zealand Vegetation. Acta Geobotanica 4: 1-31.

Santos, N.D. \& Costa, D.P. 2008. A importância de Reservas Particulares do Patrimônio Natural para a conservação da brioflora da Mata Atlântica: um estudo em El Nagual, Magé, RJ, Brasil. Acta Botanica Brasilica 22: 359-372.

Schuster, R.M. 1980. The Hepaticae and Anthocerotae of North America, v. 4. Columbia University Press, New York.

Schuster, R.M. 1992. The Hepaticae and Anthocerotae of North America, v. 4. Field Museum of Natural History, Chicago.

Sharp, A.J., Crum, H. \& Eckel, P.M. 1994. The moss flora of Mexico. Memoirs of the New York Botanical Garden 69: 1-1113.

Souza, M.J.N. 1989. Geomorfologia. In: M.L.R. Martins (coord.). Atlas do Ceará. IPLANCE, Fortaleza, pp. 14-15.

Tabarelli, M. \& Cardoso-Silva, J.M. 2002. Áreas e ações prioritárias para a conservação, utilização sustentável e repartição de benefícios da biodiversidade do bioma Caatinga. In: E.L. Araújo, A.N. Moura, E.V.S.B. Sampaio, L.M.S. Gestinari \& J.M.T. Carneiro (eds.). Biodiversidade, conservação e uso sustentável da flora do Brasil. Universidade Federal Rural de Pernambuco, Imprensa Universitária, Recife, pp. 27-30.

Valente, E.B. \& Pôrto, K.C. 2006. Hepáticas (Marchantiophyta) de um fragmento de Mata Atlântica na Serra da Jibóia, Município de Santa Teresinha, BA, Brasil. Acta Botanica Brasilica 20: 433-441.
Valente, E.B., Pôrto, K.C., Vilas Bôas-Bastos, S.B. \& Bastos, C.J.P. 2009. Musgos (Bryophyta) de um fragmento de Mata Atlântica na Serra da Jibóia, Município de Santa Terezinha, BA, Brasil. Acta Botanica Brasilica 23: 369-375.

Vilas Bôas-Bastos, S.B. \& Bastos, C.J.P. 2009. Musgos pleurocárpicos dos fragmentos de Mata Atlântica da Reserva Ecológica da Michelin, Município de Igrapiúna, BA, Brasil. II - Hypnales (Bryophyta: Bryopsida). Acta Botanica Brasilica 23: 630-643.

Visnadi, S.R. 2002. Meteoriaceae (Bryophyta) da Mata Atlântica do Estado de São Paulo. Hoehnea 29: 159-187.

Visnadi, S.R. 2005. Brioflora da Mata Atlântica do Estado de São Paulo: região norte. Hoehnea 32: 215-231.

Yano, O. 1981. A checklist of Brazilian mosses. The Journal of Hattori Botanical Laboratory 50: 279-456.

Yano, O. 1984a. Briófitas. In: O. Fidalgo \& V.L.R. Bononi (eds.). Técnicas de coleta, preservação e herborização de material botânico. Instituto de Botânica, São Paulo, pp. 27-30.

Yano, O. 1984b. Checklist of Brazillian liverworts and hornworts. The Journal of the Hattori Botanical Laboratory 56: 481-548.

Yano, O. 1989. An additional checklist of Brazilian bryophytes. The Journal of Hattori Botanical Laboratory 66: 371-434.

Yano, O. 1995. A new additional annotated checklist of Brazilian bryophytes. The Journal of Hattori Botanical Laboratory 78: 137-182.

Yano, O. 2006. Novas adições ao catálogo de Briófitas Brasileiras. Boletim do Instituto de Botânica 17: 1-142.

Yano, O. 2008. Catálogo de Antóceros e Hepáticas brasileiros: literatura original, basiônimo, localidade-tipo e distribuição geográfica. Boletim do Instituto de Botânica 19: 1-110.

Yano, O. 2010. Levantamento de novas ocorrências de briófitas brasileiras. Instituto de Botânica. http://www. ibot.sp.gov.br (acesso em 15.05.2011).

Yano, O. \& Peralta, D.F. 2007. Musgos (Bryophyta). In: J.A. Rizzo (coord.). Flora dos Estados de Goiás e Tocantins: Criptógamos, v. 6. Universidade Federal de Goiás, Goiânia.

Yano, O. \& Pôrto, K.C. 2006. Diversidade das briófitas das Matas Serranas do Ceará, Brasil. Hoehnea 33: 7-40.

Yano, O., Bordin, J. \& Peralta, D.P. 2009. Briófitas dos Estados do Ceará, Maranhão, Paraíba, Piauí e Rio Grande do Norte (Brasil). Hoehnea 36: 387-415.

Yuzawa, Y. 1991. A monograph of subgen. Chonanthelia of genus Frullania. Journal of the Hattori Botanical Laboratory 70: 181-291.

Zartman, C.E. \& Ilkiu-Borges, A.L. 2007. Guia para briófitas epífilas da Amazônia Central. Instituto Nacional de Pesquisas da Amazônia, Manaus. 